\title{
Improvement in Waldenström's Macroglobulinemia after Successful Treatment of HCV with Direct-acting Antivirals
}

\author{
Mattia Crespi, ${ }^{\star}$ Maria Giulia Demarzo, ${ }^{*}$ Matteo Brunacci, ${ }^{*}$ Gaia Pellegatta, ${ }^{*}$ \\ Fabio Ferrando, ${ }^{* *}$ Alberto Ballestrero, ${ }^{* *}$ Federica Grillo, ${ }^{* * *}$ Vincenzo Savarino, ${ }^{*}$ Edoardo G. Giannini ${ }^{*}$
}

* Gastroenterology Unit, Ospedale Policlinico San Martino, IRCCS per l'Oncologia, University of Genoa, Genoa, Italy. ** Internal Medicine and Oncology Unit, Department of Internal Medicine, Ospedale Policlinico San Martino, IRCCS per l'Oncologia, University of Genoa, Genoa, Italy. ${ }^{\star * \star}$ Pathology Unit, Department of Surgical Sciences, Ospedale Policlinico San Martino, IRCCS per I'Oncologia, University of Genoa, Genoa, Italy.

\section{ABSTRACT}

Chronic hepatitis C (HCV) virus infection may be associated with several non-hepatic manifestations, mainly driven by chronic immune stimulation, such as mixed cryoglobulinemia and Non-Hodgkin's Lymphoma. This association has been proved by several meta-analyses and some interventional studies demonstrating that antiviral treatment may be effective in inducing $\mathrm{HCV}$-associated lymphoma regression. The recent advent of direct acting antivirals (DAAs) in the therapeutic armamentarium of HCV infection made possible treatment of patients with advanced liver disease. Here we report on a rare association of a cirrhotic patient with HCV and Waldenström's Macroglobulinemia with severe cryoglobulinemia, who had already failed an interferon-based antiviral regimen, whose haematologic disease was ameliorated by HCV eradication following treatment with sofosbuvir and simeprevir with ribavirin, and where successful treatment was accompanied also by consistent improvement in liver function and parameters of portal hypertension.

Key words. Cirrhosis. Portal hypertension. Outcome. Haematology.

\section{BACKGROUND}

Hepatitis C virus (HCV) infects more than 184 million people worldwide, and sequelae of chronic infection such as chronic hepatitis, cirrhosis, and hepatocellular carcinoma represent a major public health problem. ${ }^{1}$ The recent development of direct-acting antiviral agents (DAAs) for the treatment of chronic HCV infection in adults-in particular second generation DAA that made possible interferon (IFN)-free therapy - has revolutionized the treatment of HCV infection allowing for safe treatment of patients with advanced disease with unprecedented high rates of sustained virological response (SVR). ${ }^{2}$ It is projected that by 2030 chronic HCV infection should theoretically be confined, but even after few years from the introduction of DAAs the landscape of patients with HCV infection and advanced disease has dramatically changed, with a chance of delisting patients due to improved liver function, and a decline in listing for liver transplantation and occurrence of hepatocellular carcinoma. ${ }^{3-6}$ Moreover,
HCV infection has several non-hepatic manifestations, mainly driven by chronic immune stimulation, such as mixed cryoglobulinemia and Non-Hodgkin's Lymphoma (NHL). ${ }^{7}$ This association has been proved by several meta-analyses and some interventional studies demonstrating that antiviral treatment with pegylated-IFN or DAAs, in monotherapy or in combination with ribavirin, is effective in inducing $\mathrm{HCV}$-associated lymphoma regression. ${ }^{7-9}$ As a result, the antiviral treatment has been proposed as first line therapy in asymptomatic patients with HCV- related indolent NHL who do not need immediate conventional treatment. Despite a potential additive effect inherent to its immune-modulatory properties, IFN treatment has a low SVR rate in patients with cirrhosis and is burdened by several side effects, being not recommended in patients with advanced disease; therefore, DAAs therapy is the treatment of choice in these patients due to a better profile of safety and efficacy.

Among B-cell NHL, the main subtypes associated with $\mathrm{HCV}$ are marginal zone lymphoma, diffuse large B-cell 
lymphoma, and lymphoplasmacytic lymphoma. ${ }^{10}$ Waldenström's Macroglobulinemia (WM) is a lymphoplasmacytic lymphoma characterised by the production of monoclonal IgM involving bone marrow, and there is evidence for an association between HCV infection and WM, with a 3-fold higher risk of this lymphoproliferative disorder in HCV-positive subjects as compared to controls. ${ }^{10,11}$ Although the evidence for this association is nevertheless debated and data on optimal treatment of patients affected by WM with concurrent HCV infection are limited, there are reports of antiviral treatment in these patients. ${ }^{12}$ At the same time, however, the downstream consequences of clinical features of WM such as cryoglobulinemia-induced renal damage, as well as the side effects of drugs used to treat the underlying haematologic malignancy (e.g., steroids, anti-CD 20), may hamper successful antiviral treatment.

This case reports on a middle-aged lady with chronic HCV infection, cirrhosis, and WM. In this case, the course of disease was worsened by severe renal damage due to cryoglobulinemia that required rituximab and steroids treatment; the patient was unsuccessfully treated with an IFN-based therapy in the pre-DAAs era, and was later retreated with DAAs obtaining SVR and showing, besides improvement in liver function, also a dramatic improvement in clinical and biochemical manifestations of WM.

\section{CASE PRESENTATION}

A 50-year-old Caucasian lady was referred to our Gastroenterology Unit in 2010 for assessment of chronic HCV infection (genotype 1b) and cirrhosis.

Medical history included hemicolectomy for congenital megacolon at the age of 20 , cholecystectomy for biliary lithiasis, and steroid-induced diabetes. The patient was referred to our Unit from the Haemato-Oncology Unit of the Internal Medicine Department where, in 2009, she was diagnosed with WM (monoclonal component: 36-43 g/L) by bone marrow biopsy (Figure 1). The haematologic malignancy was associated with mixed cryoglobulinemia (cryocrite: $89 \%$ ) and vasculitis. At diagnosis, due to the presence of renal damage, the patient underwent kidney biopsy with evidence of membranoproliferative glomerulonephritis. At the same time, the patient complained of symptoms compatible with peripheral neuropathy (sensitive and motor deficit). A first cycle of rituximab was administered in November 2009, with a mild clinical response (improvement of vasculitis-related peripheral neuropathy) but with no monoclonal component reduction. When the patient was referred to our Unit, clinical and biochemical work-up was compatible with compensated cirrhosis without clinically relevant portal hypertension, and a 48-week course of antiviral treatment with pegylated-IFN $\alpha 2_{\mathrm{a}}(135 \mu \mathrm{g} /$ weekly $)$ with low-dose ribavirin $(200 \mathrm{mg} /$ daily) due to reduced glomerular filtration rate, was started. Antiviral treatment, besides being burdened by the common side effects of IFN and ribavirin (anaemia, fatigue, flu-like symptoms) and despite erythropoietin support, led to a worsening of peripheral neuropathy (need for crutches) that greatly affected the patient's quality of life. This notwithstanding, the patient was able to complete the whole course of antiviral treatment, showing negativity of serum HCV RNA during therapy, but with reappearance of viraemia at the end of treatment (relapse). In December 2012 the patient was admitted to hospital because of bilateral feet ischaemia, with instrumental evidence of severe peripheral vasoconstriction as the cause of symptoms, for which she was treated with in-
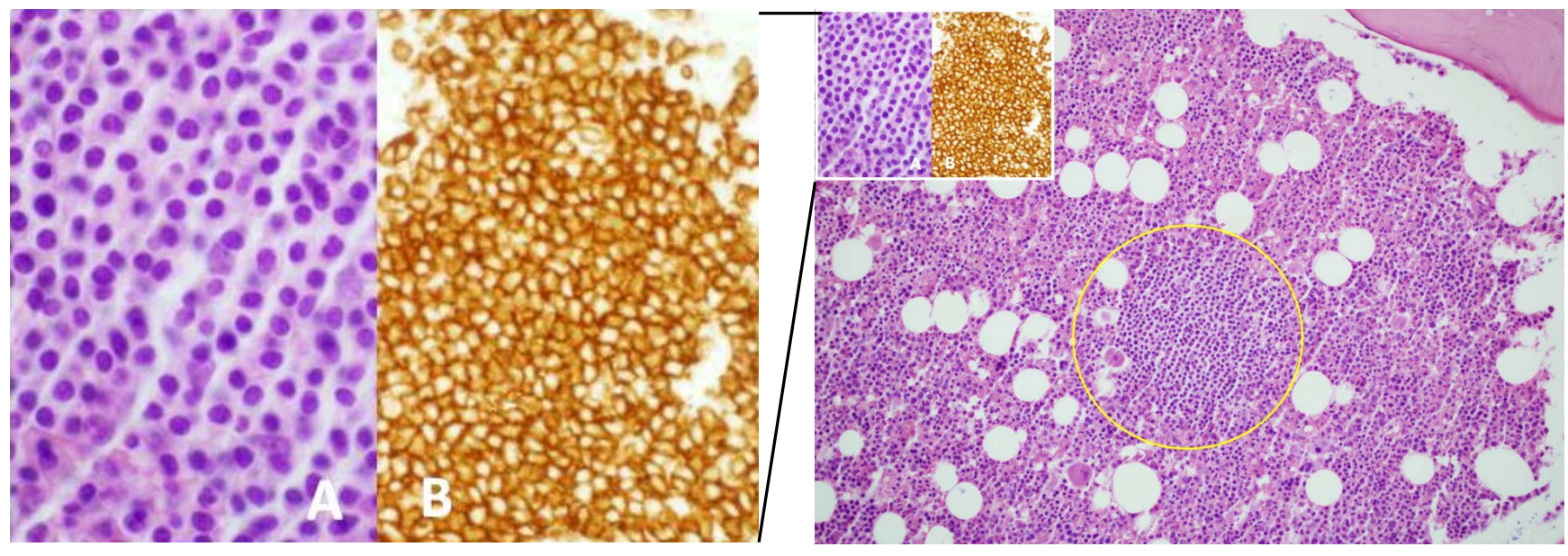

Figure 1. Bone marrow biopsy showing focale intertrabecular infiltration of lymphoplasmacytic cells (yellow circle) with plasmocytoid features (see inset $A$ ) and expression of CD20 (see inset B). Haematoxylin and Eosin, $x 20$ magnification, main figure; Haematoxylin and Eosin, x60 magnification, inset $A ; C D 20, x 60$ magnification, inset $B$. 
A. Monoclonal component ( $\mathrm{g} / \mathrm{L})$.

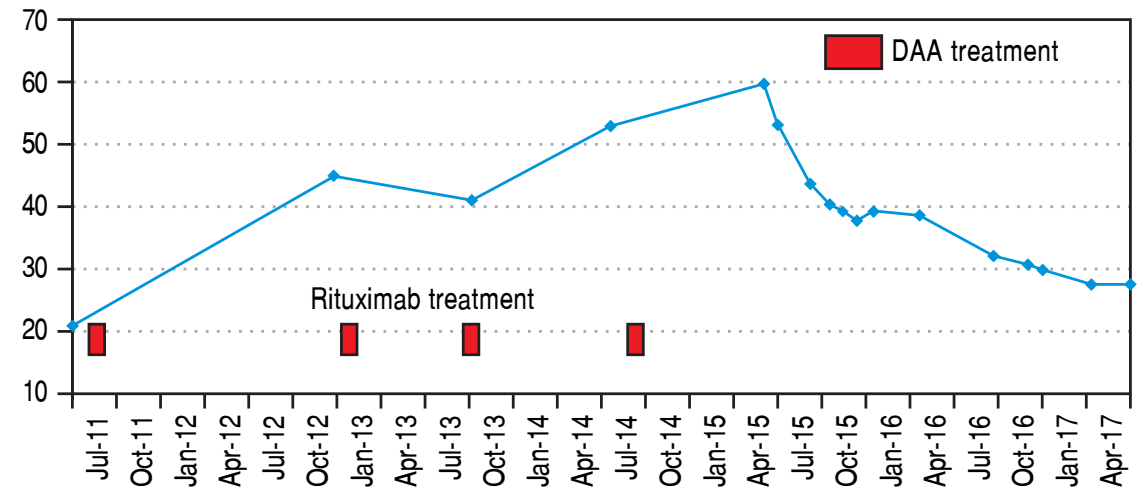

B.P-lgM (g/L).



Figure 2. $P$ - $\lg M(g / L)$. Monoclonal component $(g / L)$. Modifications in monoclonal component (A) and plasmatic lgM (B) during the course of the disease.

travenous iloprost and metilprednisolone. Moreover, due to the absence of other therapeutic options for HCV infection and the presence of symptoms, the patient underwent three more cycles of rituximab until May 2015, although she experienced no significant haematologic and clinical improvement.

In June 2015, when IFN-free DAA treatment for HCV became available in our Unit, a 12-week course of sofosbuvir (400 mg/day), simeprevir (150 mg/day), and ribavirin (400 mg/day) was started; before starting DAAs treatment her laboratory work-up showed a monoclonal component of $53.4 \mathrm{~g} / \mathrm{L}$ and quantitative plasmatic $\operatorname{IgM}=$ $71.3 \mathrm{~g} / \mathrm{L}$. During antiviral treatment, the patient became HCV RNA negative at week 4 (lower limit of detection: $15 \mathrm{IU} / \mathrm{mL}$ ), and her symptoms greatly improved: in particular, she reported a dramatic improvement in fatigue and peripheral neurologic symptoms, a finding that she had not experienced during the IFN course of treatment despite transient HCV RNA negativity. HCV RNA negativity persisted throughout the whole DAAs treatment as well as after treatment cessation, thus resulting in the achievement of SVR12. Contemporaneously, we observed a biochemical improvement that consisted in a progressive and steady decrease in the monoclonal component (nadir: $27.3 \mathrm{~g} / \mathrm{L}$, Figure 2A) and plasmatic IgM (41.5 g/L, Figure 2B) that lingered on even after treatment cessation and during follow-up. Bence-Jones protein in urine became negative. Moreover, we also observed consistently satisfying haemoglobin levels despite ribavirin treatment (median haemoglobin during treatment: $10.1 \mathrm{~g} / \mathrm{dL}$ ), and the patient did not require erythropoietin support any more. Lastly, as far as indexes related to liver function and indirect parameters suggestive of portal hypertension are concerned, albumin serum levels improved (from $27.7 \mathrm{~g} / \mathrm{L}$ to $40.3 \mathrm{~g} / \mathrm{L}$, Figure $3 \mathrm{~A}$ ) and platelet counts increased (from $146 \times 109 / \mathrm{L}$ to $176 \times 109 / \mathrm{L}$, Figure 3B) both during and after treatment; moreover, transient hepatic elastography measurement assessed before treatment and at post-treatment follow-up showed a marked improvement, from a pretreatment value of $26 \mathrm{KPa}$ to a follow-up value of $17 \mathrm{KPa}$.

\section{DISCUSSION}

HCV infection is known to be associated with haematological manifestations such as mixed cryoglobulinemia and B-cell NHL, and several pathogenic hypotheses support this association. In particular, it is either hypothesized that chronic antigen stimulation of B-cells may 
A

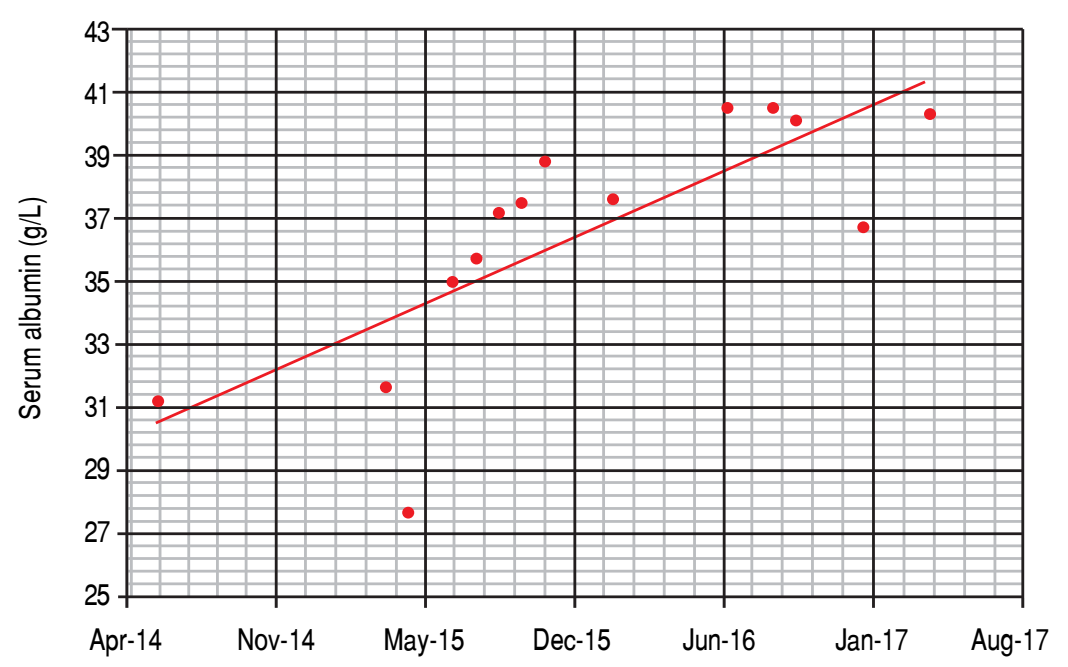

B

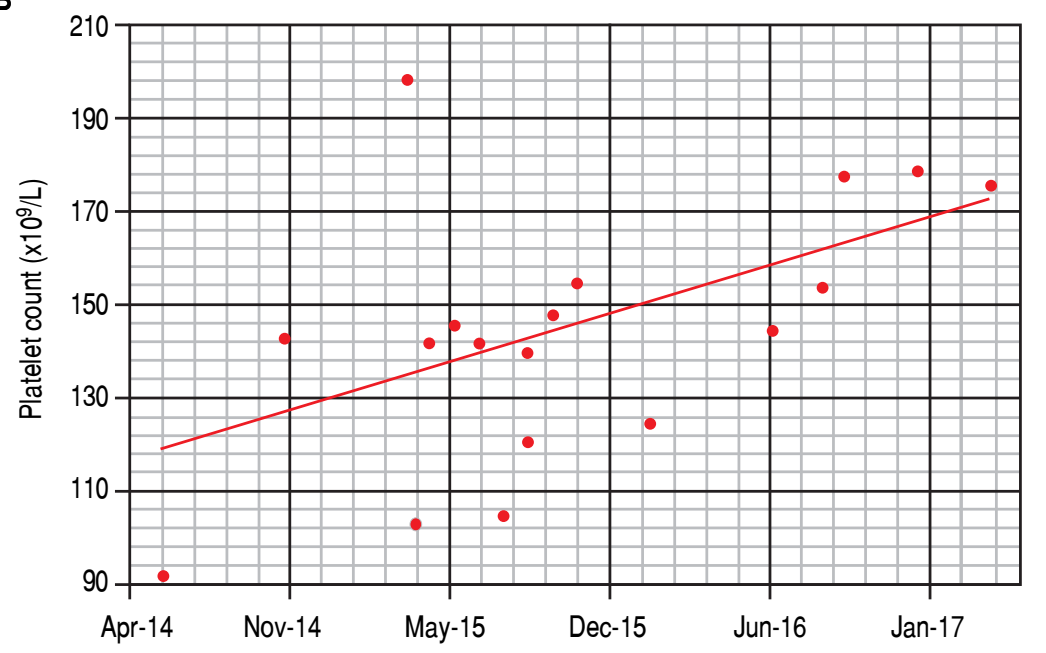

Figure 3. Modifications in serum albumin (A) and platelet count $(B)$ during the course of the disease. drive their uncontrolled proliferation, or that oncogenic effects in B cells may be mediated by intracellular viral proteins, or lastly that chronic inflammation due to HCV infection may induce mutations in B-lymphocytes through the generation of reactive oxygen species. ${ }^{13}$ Chemotherapy alone often does not guarantee a clinical and biochemical improvement, especially for indolent lymphomas and those without large nodal or extranodal masses, while successful antiviral therapy reportedly improved, besides liver condition, also characteristics of the associated haematologic disease. A recent meta-analysis reported a 73\% lymphoma response rate after IFN-based antiviral therapy in patients with chronic HCV infection, with histological type as the main predictive response factor. ${ }^{14}$ In particular, marginal zone lymphomas achieved higher response rates (i.e., up to $81 \%$ ), whereas small lym- phocytic lymphomas - that are not epidemiologically linked with HCV infection - showed no significant improvement despite virus clearance. ${ }^{8,15}$ As far as the association between WM and HCV infection is concerned, there is evidence that the presence of chronic infection with $\mathrm{HCV}$ increases the risk of MW, although in the literature there are studies reporting contrasting results and denying this association. ${ }^{10,12,16}$ Moreover, although haematological disease improvement after virus eradication in patients with lymphoproliferative disorders has been documented in case reports, cohort studies, and systematic reviews, there is scanty evidence of improvement in WM after HCV eradication. ${ }^{7,9,14}$ Lastly, there is even a recent report of severe myelosuppression potentally associated with DAAs treatment in a patient with HCV infection and lymphoplasmocytic lymphoma. ${ }^{17}$ Indeed, there are few exist- 
ing case reports describing IFN $\alpha$ and ribavirin treatment of HCV-associated cryoglobulinemia and WM, although to the best of our knowledge none of them using an IFNfree therapy. $8,12,18$

In this report, we have described the case of a patient with HCV-related cirrhosis with associated cryoglobulinemia and WM, and who was treated with multiple rituximab cycles. While anti-CD20 treatment minimally modified the course of the disease, and IFN-based antiviral treatment with $\mathrm{HCV}$ was difficult to tolerate and unsuccessful, HCV eradication with IFN-free treatment led to a dramatic clinical and biochemical improvement in her haematological condition, besides ameliorating liver function as well as liver stiffness. Because of the rarity of WM and the lack of consensus regarding its association with $\mathrm{HCV}$, the current guidelines for the management of WM do not contemplate HCV eradication among the potential therapeutic options. ${ }^{19}$ Here we report how HCV clearance was clearly associated with a progressive and consistent improvement of liver disease; DAAs treatment was well-tolerated and led to SVR in a difficult-to-treat patients characterised by several negative prognostic factors for SVR such as advanced liver disease, diabetes, renal damage, and previous unsuccessful antiviral treatment. In particular, although liver disease stabilization - or even improvement - though not guaranteed was somehow expected on the basis of the results of studies carried out in patients with advanced disease, the noteworthy improvement in haematological parameters was quite unexpected, also taking into account previous negative reports in a similar setting. ${ }^{2,4,17}$ In particular, we observed an improvement in liver synthetic function and a decrease in parameters associated with portal hypertension such as platelet count and liver stiffness; although the improvement in platelet count might have been related to the concomitant improvement of the haematological disease, and platelet count may have a fluctuating course in patients with cirrhosis, we feel that this consistent result associated with the improvement in albumin serum levels may represent and indirect evidence of actual amelioration of liver disease, despite we did not measure thrombopoietin serum levels. ${ }^{20}$ Furthermore, we could not indisputably attribute the decrease in liver stiffness that we observed in this patient solely to an improvement in liver fibrosis and potentially to a decrease in portal hypertension, as we did not perform a liver biopsy and did not measure portal pressure; however, the global interpretation of the findings we observed, together with recent evidence from the literature, seems to suggest that liver stiffness improvement after DAAs treatment may derive not only from aminotransferase normalization and necro-inflammatory activity decrease, but also to improvement in liver dis- ease stage, and the durability of response after antiviral therapy in HCV patients calls for future studies addressing this issue. ${ }^{21,22}$

To conclude, we feel that further investigations are needed to compare the efficacy of HCV eradication to existing therapies for WM so as to develop a solid combined strategy, while we strongly suggest that these cases should be managed in the context of a multidisciplinary approach that includes hepatologists and haematologists with the aim to pursue the best management for the patient.

\section{ABBREVIATIONS}

- DAAs: direct-acting antiviral agents.

- HCV: hepatitis C virus.

- IFN: interferon.

- NHL: Non-Hodgkin's lymphoma.

- SVR: sustained virological response.

- WM: Waldenström's macroglobulinemia.

\section{FUNDING ROLE}

This manuscript was not supported by any funding.

\section{DECLARATION OF CONFLICT OF INTEREST}

The authors declares that there is no conflict of interest regarding the publication of this article.

\section{REFERENCES}

1.Thrift AP, El-Serag HB, Kanwal F. Global epidemiology and burden of HCV infection and HCV-related disease. Nat ReV Gastroenterol Hepatol 2017; 14: 122-32.

2.Petta S, Marzioni M, Russo P, Aghemo A, Alberti A, Ascione $A$, Antinori A, et al. Ombitasvir, paritaprevir, and ritonavir, with or without dasabuvir, plus ribavirin for patients with hepatitis $C$ virus genotype 1 or 4 infection with cirrhosis (ABACUS): a prospective observational study. Lancet Gastroenterol Hepatol 2017; 2: 427-34.

3.European Union HCV Collaborators. Hepatitis C virus prevalence and level of intervention required to achieve the $\mathrm{WHO}$ targets for elimination in the European Union by 2030: a modelling study. Lancet Gastroenterol Hepatol 2017; 2: 325-36.

4.Belli LS, Berenguer M, Cortesi PA, Strazzabosco M, Rockenschaub SR, Martini S, Morelli C, et al. Delisting of liver transplant candidates with chronic hepatitis $C$ after viral eradication: A European study. J Hepatol 2016; 65: 524-31.

5.Flemming JA, Kim WR, Brosgart CL, Terrault NA. Reduction in liver transplant wait-listing in the era of direct-acting antiviral therapy. Hepatology 2017; 65: 804-12.

6.Petta S, Cabibbo G, Barbara M, Attardo S, Bucci L, Farinati F, Giannini EG, et al. Hepatocellular carcinoma recurrence in patients with curative resection or ablation: impact of $\mathrm{HCV}$ eradication does not depend on the use of interferon. Aliment Pharmacol Ther 2017; 45: 160-8.

7.Arcaini L, Merli M, Volpetti S, Rattotti S, Gotti M, Zaja F. Indolent B-cell lymphomas associated with $\mathrm{HCV}$ infection: clinical 
and virological features and role of antiviral therapy. Clin Dev Immunol 2012; 2012: 638185.

8.Arcaini L, Besson C, Frigeni M, Fontaine H, Goldaniga M, Casato $M$, Visentini $M$, et al. Interferon-free antiviral treatment in B-cell lymphoproliferative disorders associated with hepatitis C virus infection. Blood 2016; 128: 2527-32.

8.Gisbert JP, Garcia-Buey L, Pajares JM, Moreno-Otero R. Prevalence of hepatitis $C$ virus infection in B-cell non-Hodgkin's lymphoma: systematic review and meta-analysis. Gastroenterology 2003; 125: 1723-32.

9.Merli M, Carli G, Arcaini L, Visco C. Antiviral therapy of hepatitis $\mathrm{C}$ as curative treatment of indolent B-cell lymphoma. World J Gastroenterol 2016; 22: 8447-58.

10.Giordano TP, Henderon L, Landgren O, Chiao EY, Kramer JR, El-Serag $\mathrm{H}$, Engels EA. Risk of non-Hodgkin lymphoma and lymphoproliferative precursor diseases in US veterans with hepatitis C virus. JAMA 2017; 297: 2010-17.

11.de Sanjose S, Benavente $Y$, Vajdic CM, Engels EA, Morton LM, Bracci PM, Spinelli JJ, et al. Hepatitis $C$ and non-Hodgkin lymphoma among 4784 cases and 6269 controls from the International Lymphoma Epidemiology Consortium. Clin Gastroenterol Hepatol 2008; 6: 451-8.

12.Nipp R, Mitchell A, Pishko A, Metjian A. Waldenström Macroglobulinemia in hepatitis $C$ : case report and review of the current literature. Case Rep Oncol Med 2014; 2014: 165670.

13.Mihaila RG. Hepatitis C virus - associated B cell non-Hodgkin's lymphoma. World J Gastroenterol 2016; 22: 6214-23.

14.Peveling-Oberhag J, Arcaini L, Bankov K, Zeuzem S, Herrmann $\mathrm{E}$. The anti-lymphoma activity of antiviral therapy in HCV-associated B-cell non-Hodgkin lymphomas: a metaanalysis. J Viral Hep 2016; 23: 536-44.

15.Slager SL, Benavente Y, Blair A, Vermeulen R, Cerhan JR, Costantini AS, Monnereau A, et al. Medical history, lifestyle, family history, and occupational risk factors for chronic lymphocytic leukemia/small lymphocytic lymphoma: the InterLymph Non-Hodgkin Lymphoma Subtypes Project. J NatI Cancer Inst Monogr 2014; 2014: 41-51.

16.Leleu X, O'Connor K, Ho AW, Santos DD, Manning R, Xu L, Hatjiharissi $E$, et al. Hepatitis $C$ viral infection is not associat- ed with Waldenström's macroglobulinemia. Am J Hematol 2007; 82: 83-4.

17.Senín A, Broquetas T, Cañete N, Lens S, Londoño MC, Ferraro $\mathrm{M}$, Forns $\mathrm{X}$, et al. Aplastic anemia and severe myelosuppression with boceprevir or simeprevir-containing hepatitis C virus treatment. Ann Hepatol 2017; 16: 312-17.

18.Álvarez-Ruiz SB, García-Río I, Aragüés M, Fraga J, Locertales Pueyo J, Fernández-Herrera J, García-Díez A. Leucocytoclastic vasculitis, hepatitis $C$ virus-associated mixed cryoglobulinaemia with biclonal gammopathy and Waldenström macroglobulinaemia. Br J Dermatol 2004; 151: 937-9.

19.Leblond V, Kastritis E, Advani R, Ansell SM, Buske C, Castillo JJ, García-Sanz R, et al. Treatment recommendations from the Eighth International Workshop on Waldenstrom's Macroglobulinemia. Blood 2016; 128: 1321-8.

20.Giannini EG, Peck-Radosavljevic M. Platelet dysfunction: status of thrombopoietin in thrombocytopenia associated with chronic liver failure. Semin Thromb Hemost 2015; 41: 455-61.

21.Giannini EG, Basso M, Savarino V, Picciotto A. Sustained virological response to pegylated interferon and ribavirin is maintained during long-term follow-up of chronic hepatitis $C$ patients. Aliment Pharmacol Ther 2010; 31: 502-8.

22. Kondili LA, Gaeta GB, Brunetto MR, Di Leo A, lannone A, Santantonio TA, Giammario A, et al. Incidence of DAA failure and the clinical impact of retreatment in real-life patients treated in the advanced stage of liver disease: Interim evaluations from the PITER network. PLOS ONE 2017; 12: e0185728.

Correspondence and reprint request:

Edoardo G. Giannini, M.D., Ph.D., F.A.C.G. Gastroenterology Unit, Department of Internal Medicine, University of Genoa, Viale Benedetto XV, No.6, 16132, Genoa, Italy. Tel. +39010 353 7950, Fax: +39010 3538638 E-mail: egiannini@unige.it 